\title{
PARÂMETROS DENDROMÉTRICOS E RESISTÊNCIA MECÂNICA DAS ÁRVORES DE CLONES DE Eucalyptus EM ÁREAS SUJEITAS À AÇÃO DOS VENTOS
}

\author{
DENDROMETRY PARAMETERS AND MECHANICAL RESISTANCE OF Eucalyptus CLONE \\ TREES IN AREAS SUBJECT TO WIND
}

\author{
Rafael Leite Braz ${ }^{1}$ José Tarcísio da Silva Oliveira ${ }^{2}$ Antônio Marcos Rosado ${ }^{3}$ \\ Graziela Baptista Vidaurre ${ }^{4}$ Juarez Benigno Paes ${ }^{5}$
}

\begin{abstract}
RESUMO
Este trabalho foi realizado com intuito de avaliar a resistência mecânica das árvores à quebra e sua relação com as características dendrométricas, a densidade básica e a flexão estática da madeira (Módulo de elasticidade -MOE, Módulo de ruptura - MOR e Trabalho absorvido), com a finalidade de selecionar clones mais tolerantes à ação dos ventos. A resistência mecânica das árvores à quebra foi determinada a partir da simulação do efeito do vento sobre as mesmas, obtendo-se a força necessária para quebra das árvores. Foram avaliados dez clones do híbrido Eucalyptus urophylla x Eucalyptus grandis com 30 e 35 meses de idade, nos plantios florestais em baixadas. A força média para quebrar as árvores foi de 79,00 kgf, na altura média de 2,99 m. A densidade média foi de $0,40 \mathrm{~g} / \mathrm{cm}^{3}$. O MOE, MOR e Trabalho Absorvido nos ensaios de flexão estática foram iguais a $60965 \mathrm{kgf} / \mathrm{cm}^{2}, 544 \mathrm{kgf} / \mathrm{cm}^{2}$ e $10 \mathrm{~J}$, respectivamente. O clone CNB017 apresentou os melhores resultados nos teste de resistência das árvores à quebra e nos parâmetros físicos e mecânicos avaliados, sendo o mais indicado para as áreas mais susceptíveis à ação dos ventos.
\end{abstract}

Palavras-chave: quebra das árvores; parâmetros físicos e mecânicos; Eucalyptus urophylla x Eucalyptus grandis.

\section{ABSTRACT}

This work was developed in order to evaluate the mechanical breakage of trees and its relation to the dendrometric characteristics, the wood specific gravity and bending (MOE, MOR and work to maximum load) in order to select clones more tolerant to wind action. The mechanical resistance of the trees to break was determined by the simulation of the effect of wind on them, getting the strength to break the trees. Ten clones of Eucalyptus urophylla $\mathrm{x}$ Eucalyptus grandis hybrid with 30 and 35 months in forest plantations in the lowlands were evaluated. The average force to break the trees was $79.00 \mathrm{kgf}$, the average height of $2.99 \mathrm{~m}$. The average wood density was $0.40 \mathrm{~g} / \mathrm{cm}^{3}$. MOE, MOR and work to maximum load in static bending tests were equal to $60,965 \mathrm{kgf} / \mathrm{cm}^{2}, 544 \mathrm{kgf} / \mathrm{cm}^{2}$ and10 J, respectively. The CNB017 showed the best results in the resistance of trees to break test and in the physical and mechanical parameters evaluated, being con

1 Engenheiro Florestal, Msc., Doutorando do Programa de Pós-graduação em Engenharia Florestal, Centro de Ciências Florestais e da Madeira, Universidade Federal do Paraná, Av. Pref. Lothário Meissner, 900, Campus III, Jardim Botânico, CEP 80210-170, Curitiba (PR), Brasil.rafaellbraz@yahoo.com.br

2 Engenheiro Florestal, Dr., Professor Associado do Departamento de Ciências Florestais e da Madeira, Universidade Federal do Espírito Santo, Av. Governador Lindemberg, 316, CEP 29550-000, Jerônimo Monteiro (ES), Brasil. jtsilva@npd.ufes.br

3 Engenheiro Florestal, Dr., Empresa CENIBRA - Celulose Nipo Brasileira S. A., Rod. BR 381, Km 172, CEP 35196 000, Belo Oriente (MG), Brasil. antonio.rosado@cenibra.com.br

4 Engenheira Florestal, Dr ${ }^{\mathrm{a}}$., Professora Adjunto do Departamento de Ciências Florestais e da Madeira, Universidade Federal do Espírito Santo, Av. Governador Lindemberg, 316, CEP 29550-000, Jerônimo Monteiro (ES), Brasil. grazibaptista@yahoo.com.br

5 Engenheiro Florestal, Dr., Professor Associado do Departamento de Ciências Florestais e da Madeira, Universidade Federal do Espírito Santo, Av. Governador Lindemberg, 316, CEP 29550-000, Jerônimo Monteiro (ES), Brasil. jbp2@uol.com.br

Recebido para publicação em 9/02/2012 e aceito em 26/04/2013 
sidered the most suitable clone for the areas subjected to wind breakage of trees.

Keywords: breakage of trees; physical and mechanical parameters; Eucalyptus urophylla $x$ Eucalyptus grandis.

\section{INTRODUÇÃO}

O Brasil possui características edafoclimáticas favoráveis a plantios florestais com espécies do gênero Eucalyptus como fonte de matéria-prima. A elevada produtividade, homogeneidade e potencialidade de uso múltiplos das florestas de eucalipto fazem com que haja crescimento do mercado consumidor de suprimento desta matéria-prima. A madeira de eucalipto é considerada estratégica para o setor industrial brasileiro, sendo utilizada como matéria-prima para a maioria dos produtos de origem madeireira, tais como: celulose, carvão, lenha, painéis, postes, dormentes, mourões, serrados, móveis, embalagens, entre outros outros (SILVA e XAVIER, 2006). Destaca-se pela sua importância, o setor de celulose e papel, uma vez que o país é um dos maiores produtores de celulose, com tecnologias avançadas e com necessidades constantes de ampliação da área plantada com florestas de eucalipto. A área total de florestas plantadas de eucalipto e pinus no Brasil correspondem a 6.310 .450 ha e observa-se que aproximadamente $70 \%$ das áreas com eucalipto é destinada à produção de celulose e papel (ABRAF, 2010).

A qualidade do plantio florestal se deve ao efeito da combinação da genética do material, dos tratos silviculturais e do ambiente. Como resultado dos programas de melhoramento, o rápido crescimento das árvores, a fim de obter elevada produtividade, vem contribuindo para a formação de povoamentos cada vez mais jovens, influenciando na formação das árvores, bem como nas características anatômicas, química, físicas e mecânicas da madeira. Madeiras juvenis acarretam problemas ligados à qualidade da madeira, conferindo à mesma, valores baixos de densidade e resistência mecânica (LATORRACA e ALBURQUERQUE, 2000).

Diversos povoamentos florestais destinados à obtenção de madeira para a produção de celulose, principalmente, aqueles formados por árvores jovens, em função da sua constituição, estão susceptíveis à ação dos ventos, afetando consideravelmente os plantios em diversas regiões do país. Segundo Rosado (2006), os danos florestais provocados pela ação do vento vão desde o desfolhamento e quebra de galhos até a queda ou arranque das árvores, influenciando na qualidade da madeira e na produtividade, elevando os custos da colheita, deterioração da qualidade visual e contribuindo com o aumento de erosão causado pelo solo descoberto.

Na região do Vale do Rio Doce, no Estado de Minas Gerais, são evidentes os efeitos da ação dos ventos nos povoamentos, devido à ocorrência de dois fatores que associados contribuem significativamente para os danos às florestas nesta região. O primeiro é a baixa intensidade dos ventos climatológicos (vento médios diários) que conferem resistência às árvores. $\mathrm{O}$ segundo fator é a grande ocorrência de microexplosões atmosféricas de ventos, caracterizadas pela inversão de massa de ar, tendo como consequência ventos devastadores durante o período chuvoso, que são suficientemente fortes para danificar as árvores que já estão mais vulneráveis, devido à ausência de um estimulo ambiental suficiente para conferir-lhes resistência.

De acordo com Oliveira et al. (2010), onde o vento climatológico for mais intenso, maior será a resistência das árvores, pela adaptação a um esforço constante. Os autores acrescentam que, em virtude da ação dos ventos, as árvores desenvolvem formas naturais de resistência, sendo tais alterações relacionadas à constituição do lenho e às estruturas anatômicas e químicas.

Caruzzo et al. (2004) relatam que o tipo e a extensão dos danos dependem tanto de fatores meteorológicos, tais como a intensidade e duração do vento, como também de fatores intrínsecos à floresta, tais como a densidade da distribuição, arquitetura das árvores, a idade, o tipo de solo, topografia e a resistência mecânica dos troncos. Para Metcalfe e Chalk (1989), além dos fatores genéticos, as influências ambientais também são decisivas para a resposta das características ligadas à resistência aos ventos.

A ocorrência e intensidade destes ventos afetam o desenvolvimento dos plantios e as características da madeira, interferindo significativamente em suas propriedades e no produto final, bem como em questões econômicas e no desenvolvimento de atividades em campo, acarretando preocupações a empresas do setor florestal por devastar extensas 
áreas de plantios.

Portanto, é importante que se tenha o conhecimento das características dendrométricas e da resistência mecânica das árvores, o que pode contribuir para explicar a reação das árvores de Eucalyptus, quando sujeitas à ação dos ventos. As informações da ocorrência de danos apontam para a classificação de materiais com maior ou menor resistência aos fenômenos de ventos, direcionando árvores mais tolerantes para as áreas susceptíveis à ocorrência de ventos intensos.

O presente estudo tem como objetivo determinar e comparar a resistência mecânica das árvores à quebra, com as características dendrométricas, densidade básica e flexão estática para dez clones de Eucalyptus, a fim de fornecer subsídios para o melhoramento e seleção de materiais genéticos com adaptabilidade associadas aos ventos, para a obtenção de madeiras e produto final com qualidade.

\section{MATERIAL E MÉTODOS}

Foram avaliados dez clones do híbrido Eucalyptus urophylla $\mathrm{x}$ Eucalyptus grandis, sendo oito clones com 30 meses de idade e dois com 35 meses, plantados em locais de baixada com altitude próxima a $250 \mathrm{~m}$, em espaçamento de 3,33 x 3,00 m. O material é procedente de plantios florestais da Empresa Celulose Nipo-Brasileira S.A. (CENIBRA), localizada no município de Belo Oriente, na região do Vale do Rio Doce, a Leste do Estado de Minas Gerais. A região encontra-se a $42^{\circ} 22^{\prime} 30^{\prime \prime}$ de longitude Sul e a $19^{\circ} 15^{\prime} 00^{\prime \prime}$ de latitude Oeste, com altitude, nos locais de plantio, de 230 a 500 m e tipo de clima, de acordo com Köppen, Aw - clima tropical, com inverno seco e estação chuvosa no verão, manifestando-se entre os meses de novembro a abril. A precipitação na região varia de 846 a 1478 mm, com temperatura média de $25^{\circ} \mathrm{C}$, com umidade relativa média de $67,3 \%$ e com uma velocidade média dos ventos de $3,0 \mathrm{~m} / \mathrm{s}$.

As árvores foram selecionadas de parcelas de 5 x 6 árvores, totalizando 30 árvores por clone. Optou-se por árvores eretas, com boas condições fitossanitárias, sem bifurcação e fora da área de bordadura. Foram selecionadas oito árvores com os respectivos diâmetros iguais ou próximos da média entre as trinta árvores, e avaliadas a altura total, a altura da copa, o diâmetro a altura do peito (DAP$1,30 \mathrm{~m}$ ) com o uso de trema e o peso da copa, considerando folhas e galhos, com uso de uma balança de pesagem suspensa.
Cinco árvores foram selecionadas para a realização do teste de resistência mecânica das árvores à quebra, com o intuito de simular o efeito do vento sobre as árvores.

Foi adotado o seguinte método desenvolvido pela CENIBRA (2007), adquirindo assim a força (Kgf) para quebrar as árvores (Figura 1): (i) Selecionaram-se as árvores para realização do teste e, posteriormente, puxou-as em diferentes direções (norte, sul, leste, oeste, nordeste, sudoeste, noroeste e sudoeste) ou em relação à declividade (esquerda e direita, porém, "nunca morro abaixo ou acima"). O lado para a queda das árvores foi escolhido visualmente, de acordo com a direção na qual havia a maior quantidade de árvores já quebradas no local; (ii) Determinou-se para cada árvore a posição na copa onde foi amarrada, correspondente a $85 \%$ da altura total, com auxílio de uma régua milimetrada; (iii) Com auxilio de um estilingue, foi arremessada, na altura encontrada referente aos $85 \%$ da altura total da árvore, uma esfera de chumbo preso a uma linha de nylon; (iv) Foi amarrada na extremidade da linha de nylon, uma corda de polipropileno de $5 \mathrm{~mm}$ de espessura com aproximadamente $30 \mathrm{~m}$ de comprimento, e transpôs-se o mesmo galho com auxílio da linha de nylon. Para fixar bem o nylon à corda, foi utilizada uma fita isolante, e aproveitou-se o tamanho da corda para identificar a altura de amarração (altura do local amarrado na copa); (v) Foi ancorado na base de duas árvores para garantir um ângulo de $45^{\circ}$, com auxilio de uma corda de quatro metros e uma roldana. Esta roldana apresentou uma distância equivalente a do pé da árvore ao ponto de amarração na copa; (vi) Foi ancorado em uma árvore, com auxílio de uma corda de dois metros, um dinamômetro conectado a uma roldana a uma distância mínima de três metros a mais que o comprimento total da árvore; (vii) Prendeu-se em uma árvore, com auxílio de uma corda de $2 \mathrm{~m}$, um guincho elétrico já com um cabo de aço, deixando-o mais próximo possível do nível do dinamômetro, para facilitar a puxada; (viii) Passou-se a corda amarrada na copa às roldanas e conectou-se ao o cabo de aço; (ix) Ajustou-se o dinamômetro ("zerar") e começou-se a envolver o cabo de aço em ritmo constante; (x) Tracionou-se a corda até que ocorresse a quebra da árvore. A força ficou registrada no dinamômetro. Após esse procedimento, foram anotados os valores da força final utilizada para quebrar as árvores e a altura onde ocorreu a ruptura das mesmas.

A densidade básica da madeira foi obtida com base no Método Brasileiro - MB-26 da 


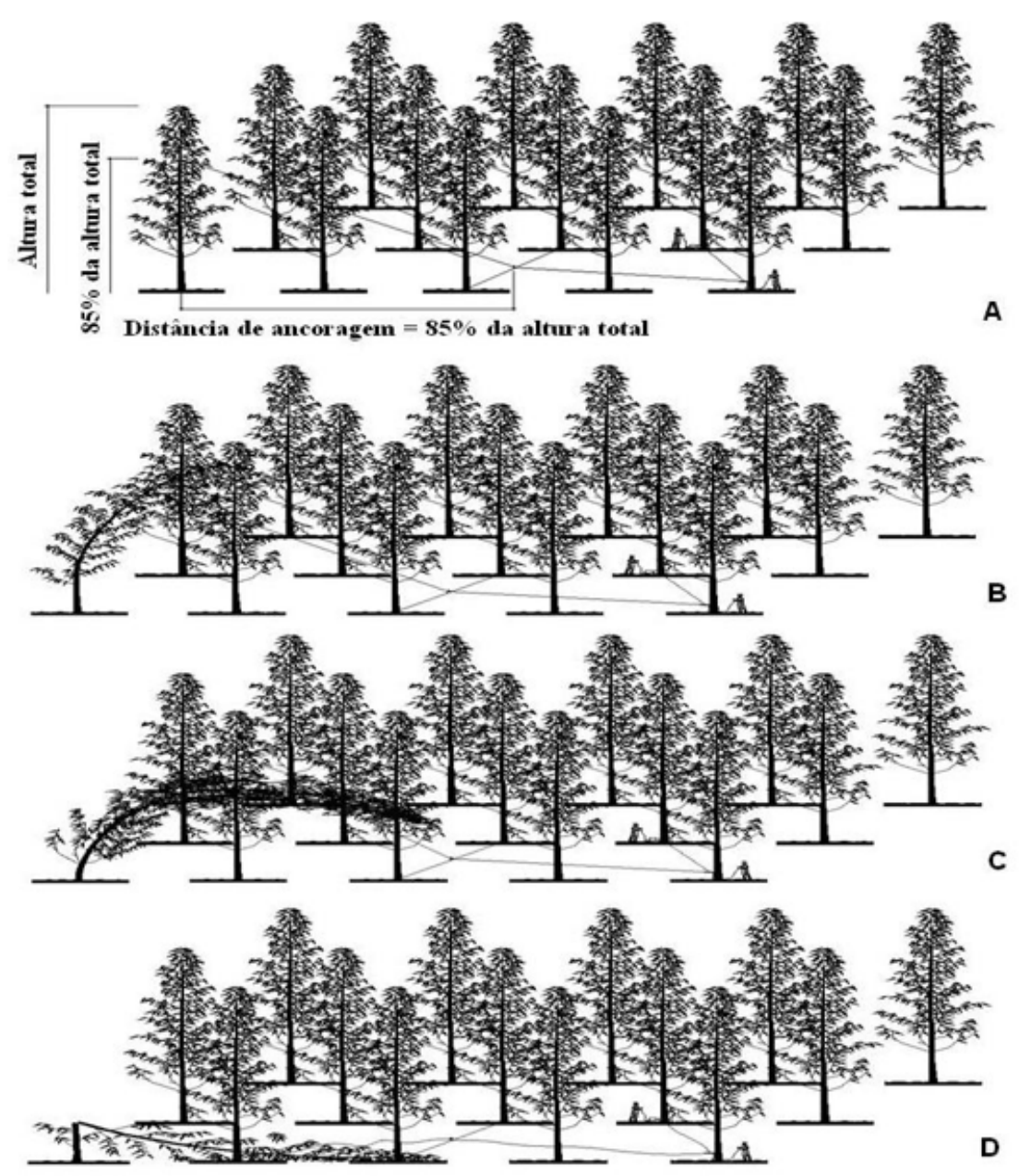

FIGURA1: Ilustração do teste de resistência das árvores à quebra: A) Instalação do teste de resistência; B) Árvore curvada; C) Árvore tombada; D) Árvore quebrada.

FIGURE 1: Ilustration of the resistance test of trees break: A) Installation of the resistance test, B) Crooked Tree, C) Fallen tree; D) Broken tree.

Associação Brasileira de Normas Técnicas - ABNT (1940). As amostras foram imersas em água até que estivessem completamente saturadas, sendo seu volume saturado determinado pelo método da balança hidrostática. Em seguida, as amostras foram secas em estufa a temperatura de $103 \pm 2^{\circ} \mathrm{C}$ até atingirem uma massa constante, determinando-a em uma balança de precisão de $0,01 \mathrm{~g}$.

A madeira para a avaliação da flexão estática foi proveniente das outras três árvores que foram abatidas e coletadas três toras por árvore, cada uma delas com $2 \mathrm{~m}$ de comprimentos (Figura 2).

As toras foram transportadas para o Laboratório de Ciência da Madeira (LCM) do Departamento de Engenharia Florestal, da Universidade Federal do Espírito Santo (UFES), no município de Jerônimo Monteiro, onde foram retirados os corpos de prova para análise da flexão estática da madeira, de acordo com o Método Brasileiro - MB-26, da Associação Brasileira de Normas Técnicas - ABNT (1940), para a determinação do Módulo de elasticidade (MOE), Módulo de ruptura (MOR) e o Trabalho absorvido. Optou-se por realizar os ensaios com os corpos de prova com a madeira na condição verde, por esta condição estar próximo às condições em campo. Os ensaios mecânicos foram executados em uma máquina universal de ensaios com sistema de aquisição de dados automatizado.

Nas análises estatísticas, os dados foram tabulados e analisados, sendo encontrados os valores de média, desvio padrão e coeficiente de variação. Para avaliar o grau de significância dos parâmetros dendrométricos, físicos e mecânico entre os clones, bem como suas variações, foi realizada uma análise 


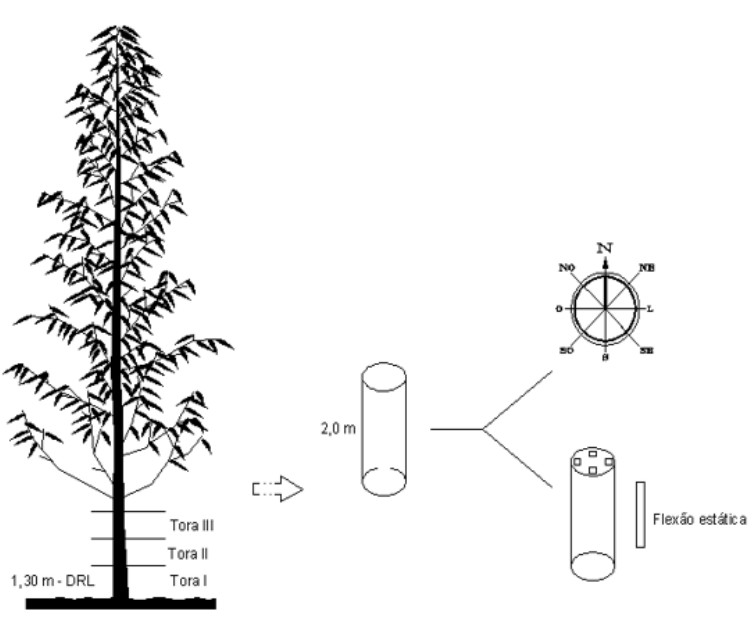

FIGURA 2 : Esquema de amostragem e localização dos corpos de prova para a flexão estática da madeira.

FIGURE 2: Schematic of sampling and location of the sample to static essay of the wood.

de variância e quando os parâmetros foram significativos, aplicou-se o teste Tukey a $5 \%$ de probabilidade para a comparação de médias. Posteriormente, foi conduzida uma análise simples da correlação entre a o teste de resistência da árvore à quebra e as variáveis de crescimento das árvores ao nível de significância de 5\%.

\section{RESULTADOS E DISCUSSÃO}

\section{Parâmetros dendrométricos e teste de resistência à quebra}

Os valores médios obtidos para as características dendrométricas e do teste de resistência mecânica das árvores à quebra para os dez clones da madeira do híbrido de Eucalyptus urophylla x Eucalyptus grandis, enocontram-se na Tabela 1.

O diâmetro médio dos clones foi de 12,34 $\mathrm{cm}$, sendo o clone CNB005 o maior diâmetro (13,34 $\mathrm{cm})$, e o clone CNB001 o menor $(10,82 \mathrm{~cm})$, mas estatisticamente iguais aos clones CNB010, CNB012 e CNB1206. A altura média das árvores dos clones foi de 17,82 m, tendo os clones CNB012, CNB1206, CNB010 e CNB005 apresentado a maior altura estatisticamente e a menor sendo observada no clone CNB008 (15,78 m). Quanto à altura de amarração, a média geral foi de $14,36 \mathrm{~m}$, tendo o clone CNB012 a maior altura $(16,58 \mathrm{~m})$ e o clone $\mathrm{CNB} 001$ a menor $(12,10 \mathrm{~m})$.

A altura média geral da copa das árvores foi de 4,96 m, sendo o clone CNB010 o que apresentou a maior altura $(5,65 \mathrm{~m})$ e o clone CNB012 a menor $(4,40 \mathrm{~m})$. O peso médio geral da copa foi de $8,09 \mathrm{~kg}$, tendo o clone CNB006 o maior peso $(10,30 \mathrm{~kg})$ e o clone CNB1206, o menor peso $(5,70 \mathrm{~kg})$.

TABELA 1: Valores médios dos parâmetros dendrométricos e da força de resistência da árvore à quebra para os dez clones do híbrido Eucalyptus urophylla $x$ Eucalyptus grandis.

TABLE 1: Average values of the dendrometric parameters and break force of the tree and breaking of the ten clones of the hybrid Eucalyptus urophylla x Eucalyptus grandis.

\begin{tabular}{cccccccc}
\hline \multirow{2}{*}{ Clone } & \multicolumn{7}{c}{ Parâmetros Avaliados } \\
\cline { 2 - 7 } & DAP(cm) & HT(m) & HC(m) & PC $(\mathrm{kg})$ & HA(m) & HQ(m) & FQ(kgf) \\
\hline CNB017 & $12,45 \mathrm{bcde}^{1}$ & $17,48 \mathrm{~b}$ & $5,31 \mathrm{a}$ & $9,55 \mathrm{ab}$ & $14,47 \mathrm{bc}$ & $3,49 \mathrm{a}$ & $98,04 \mathrm{~b}$ \\
CNB008 & $12,13 \mathrm{def}$ & $15,78 \mathrm{c}$ & $4,73 \mathrm{ab}$ & $7,40 \mathrm{ab}$ & $12,23 \mathrm{~d}$ & $3,23 \mathrm{a}$ & $65,37 \mathrm{~b}$ \\
CNB014 & $11,78 \mathrm{f}$ & $16,68 \mathrm{~b}$ & $5,19 \mathrm{a}$ & $8,40 \mathrm{ab}$ & $12,83 \mathrm{~cd}$ & $2,93 \mathrm{a}$ & $63,21 \mathrm{~b}$ \\
CNB001 & $10,82 \mathrm{~g}$ & $14,89 \mathrm{~d}$ & $3,49 \mathrm{~b}$ & $10,20 \mathrm{a}$ & $12,10 \mathrm{~d}$ & $3,61 \mathrm{a}$ & $39,76 \mathrm{~b}$ \\
CNB006 & $12,35 \mathrm{cdef}$ & $17,31 \mathrm{~b}$ & $4,31 \mathrm{ab}$ & $10,30 \mathrm{a}$ & $13,49 \mathrm{~cd}$ & $2,05 \mathrm{a}$ & $92,23 \mathrm{~b}$ \\
CNB005 & $13,34 \mathrm{a}$ & $19,24 \mathrm{a}$ & $5,62 \mathrm{a}$ & $7,20 \mathrm{ab}$ & $16,25 \mathrm{ab}$ & $1,46 \mathrm{a}$ & $196,37 \mathrm{a}$ \\
CNB010 & $13,08 \mathrm{ab}$ & $19,66 \mathrm{a}$ & $5,65 \mathrm{a}$ & $6,70 \mathrm{ab}$ & $16,14 \mathrm{ab}$ & $3,11 \mathrm{a}$ & $56,58 \mathrm{~b}$ \\
CNB007 & $11,81 \mathrm{ef}$ & $17,08 \mathrm{~b}$ & $5,52 \mathrm{a}$ & $8,20 \mathrm{ab}$ & $13,14 \mathrm{~cd}$ & $2,68 \mathrm{a}$ & $64,27 \mathrm{~b}$ \\
CNB012 & $12,80 \mathrm{abc}$ & $20,10 \mathrm{a}$ & $4,40 \mathrm{ab}$ & $7,20 \mathrm{ab}$ & $16,58 \mathrm{a}$ & $3,56 \mathrm{a}$ & $48,55 \mathrm{~b}$ \\
CNB1206 & $12,80 \mathrm{abcd}$ & $19,93 \mathrm{a}$ & $5,38 \mathrm{a}$ & $5,70 \mathrm{~b}$ & $16,33 \mathrm{a}$ & $3,79 \mathrm{a}$ & $65,63 \mathrm{~b}$ \\
\hline Média Geral & 12,34 & 17,82 & 4,96 & 8,09 & 14,36 & 2,99 & 79,00 \\
\hline CV (\%) & 6,00 & 10,25 & 14,30 & 19,02 & 12,67 & 24,92 & 56,79 \\
\hline
\end{tabular}

Em que: DAP = Diâmetro a altura do peito; HT = Altura total da árvore; HC = Altura da copa; PC = Peso da copa; $\mathrm{HA}=$ Altura de amarração; HQ = Altura da quebra; FQ = Força máxima de quebra; CV = Coeficiente de variação. ${ }^{1}$ Valores médios seguidos de mesma letra não diferem estatisticamente entre si (Tukey; $\mathrm{p}>0,05$ ). 
De modo geral, observou-se que, para algumas características, os clones diferenciaram significativamente entre si, podendo estas características influenciar na resistência das árvores quando sujeitas à ação dos ventos.

A altura média onde ocorreu a ruptura das árvores durante o teste de resistência mecânica das árvores à quebra foi de 2,99 m (Figura 3), não havendo diferença estatisticamente significativa, com o maior valor para o clone CNB1206 (3,79 m) e o menor para o clone CNB005 (1,46 m). Ferreira (2010), ao estudar quatro clones de Eucalyptus, com dois anos de idade em diferentes topografias, encontrou uma altura média de quebra de $2,5 \mathrm{~m}$, variando 1,9 a 3,1 m. Portando, pode-se observar que a altura média da quebra das árvores está próximo ao encontrado na literatura, confirmando a elevada fragilidade de quebra nesta região, quando as árvores são submetidas a esforços causados pela ação dos ventos.

A força máxima de resistência das árvores à quebra apresentou uma média geral entre os clones igual a 79,00 Kgf, tendo o clone CNB005 apresentado a maior força para quebra (196,37 Kgf), além de ter o maior diâmetro médio e a menor altura do ponto de ruptura das árvores. Já a menor foi observada no clone CNB001 (39,76 Kgf), no qual as árvores tinham os menores diâmetros e a maior altura média do ponto de ruptura. Estatisticamente, apenas o clone CNB005, diferenciou-se significativamente dos demais clones. A ordem dos clones de maior resistência das árvores à quebra para a menor ocorreu na seguinte forma: CNB005 > CNB006 > CNB017 $>\mathrm{CNB} 014>\mathrm{CNB} 007>\mathrm{CNB} 008>\mathrm{CNB} 1206$ $>$ CNB010 $>$ CNB012 $>$ CNB001. Ferreira et al.
(2010) encontraram, para a força de resistência à deformação ou à quebra, uma média entre híbridos de Eucalyptus urophylla $\times$ Eucalyptus grandis, com dois anos de idade em diferentes topografias de 69,6 kgf, variando de 59,2 a 90,0 kgf. Os autores também observaram uma tendência de força de quebra das árvores aumentarem com a inclinação do terreno.

De acordo com Rosado (2006), foi observado que existem diferenças na tolerância à ação de tempestade entre clones de Eucalyptus de mesma idade e que árvores do mesmo clone, quando plantadas em áreas de baixada, são mais suscetíveis aos danos do que quando plantadas em encosta. $\mathrm{O}$ autor ainda ressalta que fatores como inclinação do terreno e ventos climatológicos podem influenciar o desenvolvimento da árvore, promovendo a formação de lenho de reação e no desenvolvimento de tensões de crescimento no caule. Também é importante considerar que a própria arquitetura da árvore, relacionada à forma e projeção das copas, pode ser um fator preponderante no seu comportamento mecânico.

Segundo Cardoso (2011), as práticas de desrama artificial podem reduzir o impacto do vento sobre a copa, devido ao menor volume da mesma. O mesmo autor concluiu que a aplicação da desrama artificial ao nível de $50 \%$ contribuiu para o aumento da resistência à quebra pelo vento, implicando diretamente na redução da perda de hectares em florestas clonais de Eucalyptus urophylla $\mathrm{x}$ Eucalyptus grandis. A desrama artificial também contribui para a obtenção de uma madeira limpa, livre de nós, o que pode influenciar nas condições de resistência das árvores nos plantios florestais, segundo Schneider et al. (1999), a presença de nós

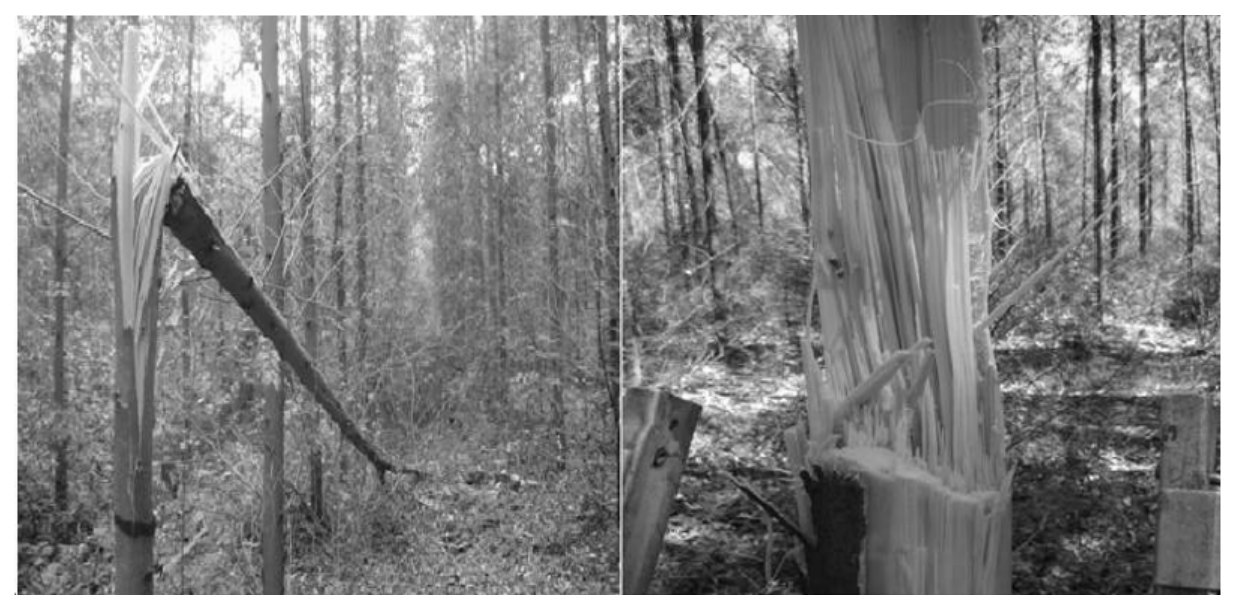

FIGURA 3: Região de ruptura das árvores durante o teste de resistência à quebra.

FIGURE 3: Region of rupture of the trees during the breaking strength test. 
vivos ou mortos, além de prejudicar a qualidade da madeira, reduz sua resistência. Melo (2004), ao estudar a intensidade de quebra das árvores em diferentes clones de Eucalyptus aos dois anos de idade decorrente da ação dos ventos, verificou que árvores mais tolerantes apresentaram maiores densidade, flexibilidade e resistência mecânica.

Os danos ocasionados por vendavais têm sido comuns em plantios comerciais de eucalipto em algumas empresas no Brasil, foram observadas variações quanto à resistência tanto ao nível de clones quanto de espécies. Dentre as espécies mais susceptíveis ao tombamento até a quebra pelo vento citam-se: o Eucalyptus pellita, Eucalyptus pilularis e Eucalyptus cloeziana (RESENDE e FANTINI JÚNIOR, 2001). Diversos autores avaliaram a resistência de espécies florestais ao vento e furacão, Thompson (1983) verificou que as espécies de Eucalyptus saligna, Eucalyptus robusta Smith, Pinus caribaea Morelet e Pinus patula Shiede et Deppe, em plantios comerciais, são mais susceptíveis aos danos por furacão, do que as espécies nativas das florestas da Jamaica. Nikles et al. (1983) verificaram uma variabilidade genética na resistência ao vento nas espécies de Pinus caribaea var. hondurensis Barr. et Golf. em Queensland, Austrália. Os autores concluíram que as espécies provenientes da região litorânea foram as mais resistentes. Nikles et al. (1998), na mesma região citada anteriormente, relataram que a espécie Acacia aulacocarpa tinha prioridade para plantios devido ao maior valor da madeira e maior resistência ao vento que a Acacia mangium. Liegel (1984) constatou-se também, em Porto Rico, que espécies de Pinus caribaea procedentes do litoral, sobreviveram a furacões com poucos danos, enquanto as procedências de Pinus oocarpa Shiede, do interior, onde furacões raramente ocorrem, ficaram severamente danificadas.

Diante do exposto, mudanças nos critérios de seleção empregados no melhoramento genético, nas práticas silviculturais e no manejo florestal adotado devem ser alcançadas, a fim de se verificar o comportamento das árvores nos diferentes povoamentos florestais, associando a diversas práticas que tendem a minimizar os danos provocados pela ação dos ventos e tempestades. Sabe-se também que propriedades da madeira podem ser influenciadas pelas características ambientais e genéticas e suas interações. Para Ferreira et al. (2010), as abordagens e as metodologias a serem empregadas permitirão o desenvolvimento no campo da interação entre árvores e ventos. Os mesmos autores ainda ressaltaram que a relação entre as propriedades da madeira e a força necessária para provocar o colapso das árvores pode contribuir para elucidar as causas das quebras das mesmas, quando expostas à ação de tempestades.

$\mathrm{Na}$ Tabela 2, são apresentadas as relações existentes entre o teste de resistência das árvores à quebra com as variáveis de crescimento da árvore, diâmetro a altura do peito, altura total e altura e peso da copa. Observa-se que os valores das correlações entre as características de crescimento e o teste de resistência das árvores à quebra foram baixos e apenas com o diâmetro foi significativa, o que indica uma fraca influência das características de crescimento sobre a resistência das árvores à quebra durante o teste. Somente as características de crescimento altura total e o diâmetro a altura do peito das árvores apresentaram uma correlação satisfatória.

Esperava-se que árvores com maiores copas tivessem maior resistência ao vento, uma

TABELA 2: Matriz dos coeficientes de correlação entre as características de crescimento da árvore e o teste de resistência das árvores à quebra.

TABLE 2: Matrix of the correlation coefficients among the growth characteristics of the tree and the the breaking strength test.

\begin{tabular}{cccccc}
\hline & FQ & DAP & HT & HC & PC \\
\hline FQ & 1 & & & & \\
DAP & $0,382^{*}$ & 1 & & & \\
HT & $0,153 \mathrm{~ns}$ & $0,799^{*}$ & 1 & & \\
HC & $0,150 \mathrm{~ns}$ & $0,411^{*}$ & $0,382^{*}$ & 1 & \\
PC & $-0,088 \mathrm{~ns}$ & $-0,260 \mathrm{~ns}$ & $-0,433^{*}$ & $-0,076 \mathrm{~ns}$ & 1 \\
\hline
\end{tabular}

Em que: $\mathrm{FQ}=$ Força máxima de quebra DAP $=$ Diâmetro a altura do peito; $\mathrm{HT}=$ Altura total da árvore; $\mathrm{HC}=$ Altura da copa; $\mathrm{PC}=$ Peso da copa; * significativo a $5 \%$ de probabilidade, ${ }^{\mathrm{ns}}$ não significativo. 
vez que maior seria a força aplicada sobre o tronco, caracterizando o efeito "vela", desempenhado pelas copas durante a ocorrência dos ventos, no entanto, não foi possível encontrar relação entre as variáveis. De acordo com Rosado (2006), geralmente as árvores mais antigas com troncos mais largos e copas mais fechadas estão mais sujeitas a sofrer danos por ventos do que as árvores mais novas, mais baixas e de troncos mais finos, devido à maior área de exposição. $\mathrm{O}$ mesmo autor ressalta que os ventos que incidem sobre as árvores exercem uma força horizontal sobre a parte exposta, gerando uma combinação de torques por torção e flexão na base da árvore. Caso esse torque seja maior que a resistência do sistema de enraizamento da árvore, ocorre o arrancamento da mesma, em outros casos, o tronco se rompe previamente e ocorre sua quebra.

Embora o grau das associações das características de crescimento com o teste de resistência das árvores à quebra resultarem em baixas correlações, as características de crescimento em função do desbaste, sítio, espaçamento, posição sociológica da árvore, entre outros fatores, estão relacionadas com a estrutura e forma do tronco, bem como a estabilidade das árvores, o que pode influenciar na resistência das árvores em áreas mais susceptíveis à ação dos ventos. Silveita et al. (1986), ao estudarem plantios via semente de Eucalyptus saligna, encontraram uma correlação alta e positiva $(0,87)$ entre a forma do tronco da árvore e a resistência ao vento. Essa correlação favorável foi também encontrada por Nikles et al. (1983), ao estudarem a resistência ao vento em procedências de Pinus caribaea var. hondurensis, em Queensland, Austrália.

$\mathrm{O}$ teste de resistência das árvores à quebra pode ser empregado como ferramenta nos programas de avaliação e seleção de clones, bem como para quantificar o problema e obter informações quanto à resistência do material, e os locais de maior ocorrência e susceptibilidade à ação dos ventos. Sugere-se que sejam realizadas avaliações da forma do tronco, uma vez que um dos critérios de seleção para o melhoramento florestal é a forma das árvores, além de avaliar as práticas silviculturais atribuídas nos plantios.

\section{Densidade básica e flexão estática}

A Tabela 3 mostra os valores médios para a Densidade básica, Módulo de elasticidade, Módulo de ruptura e Trabalho absorvido, bem com os resultados da análise do teste de média para os dez clones do híbrido Eucalyptus urophylla $\mathrm{x}$ Eucalyptus grandis.

O valor médio da densidade básica entre

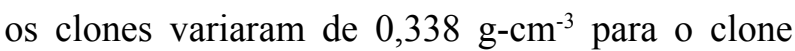

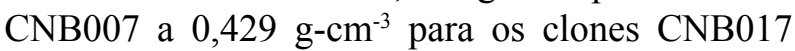

TABELA 3: Valores médios da Densidade básica, MOE, MOR e Trabalho absorvido para os dez clones do híbrido Eucalyptus urophylla $x$ Eucalyptus grandis.

TABLE 3: Average values of specific gravity, MOE, MOR and work to maximum load into the hybrid Eucalyptus urophylla $\mathrm{x}$ Eucalyptus grandis clones.

\begin{tabular}{ccccc}
\hline CLONE & Densidade Básica $\left({\left.\mathrm{g}-\mathrm{cm}^{-3}\right)}^{-3}\right.$ & MOE $\left(\mathrm{kgf}_{-} \mathrm{cm}^{-2}\right)$ & MOR $\left(\mathrm{kgf-cm}^{-2}\right)$ & Trabalho Absorvido (J) \\
\hline CNB017 & $0,429 \mathrm{a}^{1}$ & $71382 \mathrm{a}$ & $637 \mathrm{a}$ & $11 \mathrm{ab}$ \\
CNB008 & $0,394 \mathrm{bc}$ & $55136 \mathrm{c}$ & $488 \mathrm{de}$ & $7 \mathrm{~d}$ \\
CNB014 & $0,429 \mathrm{a}$ & $65063 \mathrm{ab}$ & $602 \mathrm{ab}$ & $11 \mathrm{ab}$ \\
CNB001 & $0,382 \mathrm{c}$ & $60513 \mathrm{bc}$ & $560 \mathrm{bc}$ & $10 \mathrm{bc}$ \\
CNB006 & $0,396 \mathrm{bc}$ & $59785 \mathrm{bc}$ & $530 \mathrm{~cd}$ & $10 \mathrm{bc}$ \\
CNB005 & $0,397 \mathrm{bc}$ & $60936 \mathrm{bc}$ & $561 \mathrm{bc}$ & $11 \mathrm{ab}$ \\
CNB010 & $0,369 \mathrm{c}$ & $55438 \mathrm{c}$ & $467 \mathrm{e}$ & $7 \mathrm{~d}$ \\
CNB007 & $0,338 \mathrm{~d}$ & $55541 \mathrm{c}$ & $500 \mathrm{de}$ & $8 \mathrm{~cd}$ \\
CNB012 & $0,423 \mathrm{ab}$ & $61730 \mathrm{~b}$ & $524 \mathrm{~cd}$ & $9 \mathrm{bcd}$ \\
CNB1206 & $0,396 \mathrm{bc}$ & $64122 \mathrm{~b}$ & $567 \mathrm{bc}$ & $13 \mathrm{a}$ \\
\hline Média geral & 0,400 & 60965 & 544 & 10 \\
\hline CV (\%) & 7,15 & 8,32 & 9,63 & 20,07
\end{tabular}

Em que: $\mathrm{MOE}=$ Módulo de elasticidade; MOR $=$ Módulo de ruptura; $\mathrm{CV}=$ Coeficiente de variação. ${ }^{1}$ Valores médios seguidos de mesma letra não diferem estatisticamente entre si (Tukey; $p>0,05$ ). 
e CNB014. Estatisticamente, os clones CNB017, CNB014 e o CNB012 apresentaram os maiores valores para a densidade básica da madeira.

Os valores da densidade básica da madeira foram menores que aqueles encontrados por outros autores, Mauri (2010) observou, em clones de Eucalyptus grandis $x$ Eucalyptus urophylla com aproximadamente seis anos de idade, uma densidade

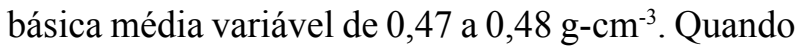
a idade era semelhante, os valores para a densidade básica da madeira foram bem próximos, Melo (2004), ao estudar clones de Eucalyptus aos dois anos de idade, encontrou uma densidade variando

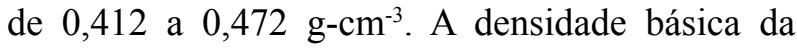
madeira é influenciada por diversos fatores e varia em função da idade, procedência, local de origem, espaçamento, da taxa de crescimento entre os gêneros e espécies e até mesmo entre as árvores e na própria árvore da mesma espécie (FERREIRA e KAGEYAMA, 1978; TOMAZELLO FILHO, 1985; SOUZA, CARPIM e BARRICHELO, 1986).

Ressalta-se que a maior tolerância das árvores nas áreas sujeitas a ventos se deve a maior densidade da madeira, em virtude da espessura maior das paredes das fibras, o que oferece maior resistência à madeira. Melo (2004), ao avaliar clones de Eucalyptus aos dois anos de idade, observou que a maior resistência à ação de tempestades está relacionada a fibras mais longas e a lumes mais estreitos.

O MOE da madeira na condição verde variou de $55.541 \mathrm{kgf}-\mathrm{cm}^{-2}$ para o clone CNB007 a $71.382 \mathrm{kgf}^{-\mathrm{cm}^{-2}}$ para o clone CNB017. Estatisticamente, o clone CNB017 apresentou o maior módulo de elasticidade diferindo dos demais clones, com exceção do clone CNB014. O módulo de elasticidade, quando elevado, indica um corpo rígido e resistente, capaz de suportar maiores esforços, o que proporciona à madeira a capacidade em voltar a sua condição original, sem alterar suas propriedades, após ser submetida a uma força. Desta forma, espera-se que os clones que apresentaram os maiores valores para MOE sejam mais tolerantes nas áreas mais expostas à ação de ventos fortes.

O MOR da madeira na condição verde variou de $467 \mathrm{kgf}-\mathrm{cm}^{-2}$ para o clone CNB010 a 637 kgf- $\mathrm{cm}^{-2}$ para o clone CNB017. Estatisticamente, o clone CNB017 apresentou o maior módulo de ruptura, diferindo dos demais clones, com exceção do clone CNB014. O módulo de ruptura demonstra os esforços mais elevados nas fibras de madeira sob influência de uma carga, o que justifica a importância do limite de resistência na ruptura em árvores sujeita à ação de ventos. Devem ser selecionados clones que apresentem maiores valores para o módulo de ruptura, uma vez que reflete a maior resistência da madeira.

Ferreira (2007), ao avaliar quatro clones de Eucalyptus, três híbridos de Eucalyptus grandis $\times$ Eucalyptus urophylla e um clone do Eucalyptus grandis com dois anos de idade, encontrou valores médios para o módulo de elasticidade, que variou de 36.597,6 $\mathrm{kgf}^{-\mathrm{cm}^{-2}}$ a 44.094,6 $\mathrm{kgf-} \mathrm{cm}^{-2}$. Os valores médios para o MOR obtidos por Melo (2004), para quatro clones de Eucalyptus com 24 meses de idade, variaram de 846,6 a $1071 \mathrm{kgf}-\mathrm{cm}^{-2}$. Segundo o mesmo autor, quanto mais elástico for o material, porém, com elevada resistência, maiores deformação poderá sofrer sem chegar ao colapso, pois não apresentará ruptura frágil.

Os valores do MOE e MOR quando comparados com outros autores, estudando materiais de idades próximas, estão em conformidade, sendo que a madeira dos dez clones avaliados apresentou baixa resistência mecânica, o que pode ser justificado pela idade.

Os valores médios para o trabalho absorvido para os dez clones estudados, com a madeira na condição verde, variaram de $7 \mathrm{~J}$ para os clones CNB008 e CNB010 a 13 J para o clone CNB1206. O clone CNB1206 apresentou o maior valor para o trabalho absorvido, seguido pelos clones CNB017, CNB005 e CNB014, diferindo-se estatisticamente do demais. O trabalho absorvido no ensaio de flexão fornece uma ideia sobre a facilidade (maior ou menor) com a qual o material irá sofrer ruptura. Maiores valores de trabalho absorvido indicam que a madeira é mais tenaz, característica desejável para que as árvores tenham maiores condições de suportar os esforços causados pelo vento.

A fim de verificar se haveria variação no tronco das árvores até os seis metros de altura, uma vez que as regiões de ruptura das árvores variavam de 1,46 a $3,75 \mathrm{~m}$ de altura, foram comparadas as toras I, II e III retiradas de cada árvore (Figura 2) dos clones avaliados. A Figura 4 mostra os valores médios do MOE, MOR e Trabalho absorvido para cada tora, bem como a comparação entre as toras nos diferentes clones, na condição de madeira verde.

Para a tora $\mathrm{I}$, os valores do MOE variaram

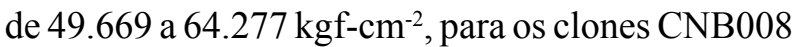
e CNB017, respectivamente. Na tora II, os valores variaram de $55.943 \mathrm{kgf}-\mathrm{cm}^{-2}$ para o clone CNB007 a $72521 \mathrm{kgf}-\mathrm{cm}^{-2}$ para o clone CNB017. Para a 

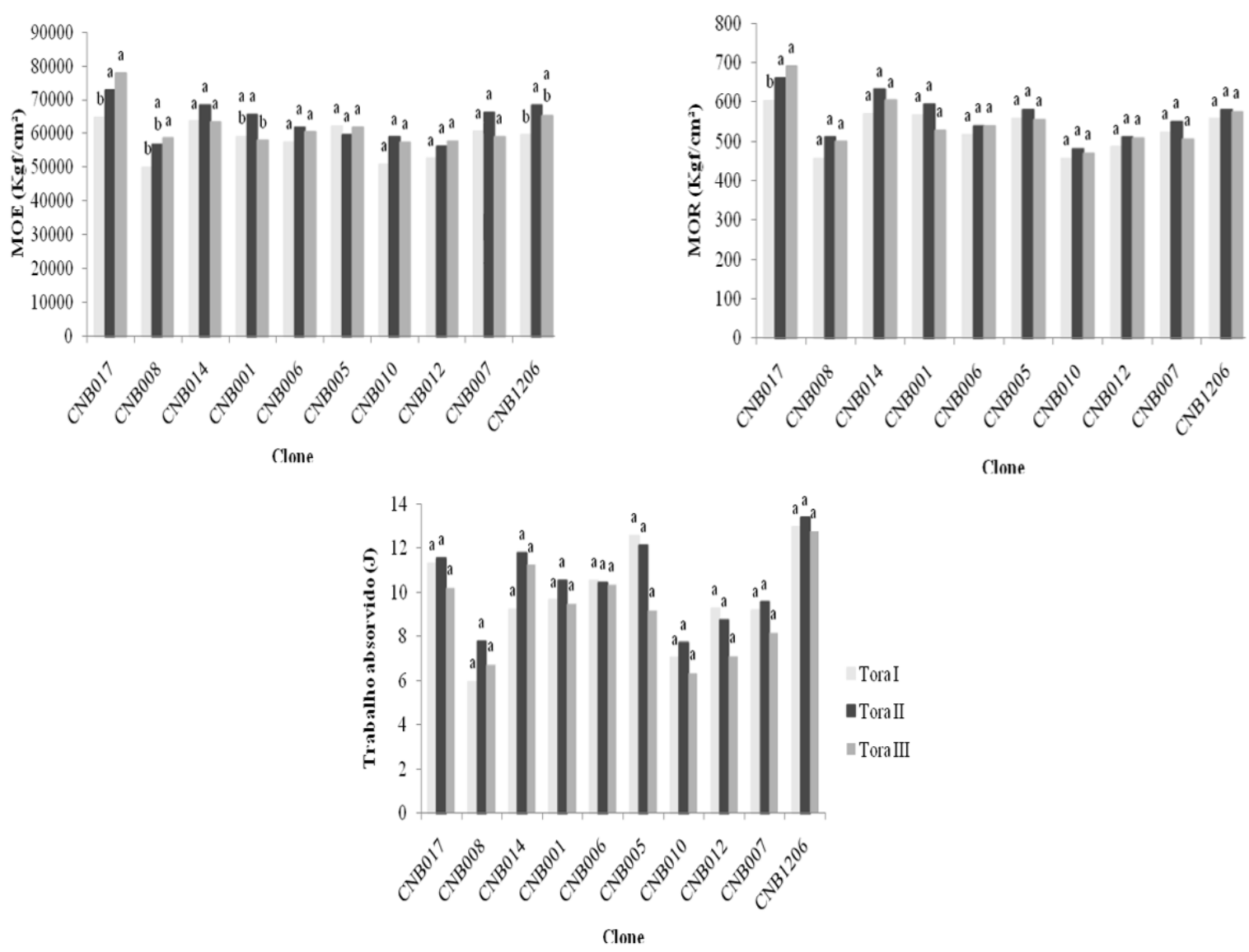

Em que: Valores médios seguidos de mesma letra não diferem estatisticamente entre si (Tukey; $p>0,05$ ).

FIGURA 4: Representação gráfica dos valores médios do MOE e MOR $\left(\mathrm{kgf}^{\left.-\mathrm{cm}^{-2}\right)}\right.$ e do Trabalho absorvido (J) para as toras I, II e III, para cada clone estudado, com a madeira na condição verde.

FIGURE 4: Graphic representation of average values of MOE and MOR $\left(\mathrm{kgf}-\mathrm{cm}^{-2}\right)$ and work to maximum (J) to the logs I, II and III, in each clone studied, with the wood in green condition.

tora III, os valores variaram de 57.065 a 78.539 kgf-cm ${ }^{-2}$, para os clones CNB010 e CNB017, respectivamente. Os clones CNB017, CNB008, CNB001 e o CNB1206 apresentaram diferença estatística entre as toras.

Os valores do MOR na tora I variaram de 454 a $600 \mathrm{Kgf}^{-\mathrm{cm}^{-2}}$ para os clones CNB008 e CNB017, respectivamente. Na tora II, os valores variaram de $478 \mathrm{kgf}^{-\mathrm{cm}^{-2}}$ para o clone CNB010 a $658 \mathrm{kgf}^{-\mathrm{cm}^{-2}}$ para o clone CNB017. Para a tora III,

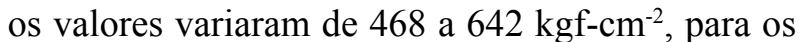
clones CNB010 e CNB017, respectivamente. São observadas diferenças estatísticas entre as toras apenas para o clone CNB017.

$\mathrm{Na}$ tora I, os valores do trabalho absorvido variaram de 6 a $13 \mathrm{~J}$ para os clones CNB008 e CNB005, respectivamente. Na tora II, os valores variaram de $8 \mathrm{~J}$ para o clone $\mathrm{CNB} 008$ a $12 \mathrm{~J}$ para $\mathrm{o}$ clone CNB014. Para a tora III, os valores variaram de 7 a $11 \mathrm{~J}$, para os clones CNB008 e CNB014, respectivamente. Conforme apresentado, não houve diferença estatística entre as toras, para os dez clones avaliados.

Embora os valores do Módulo de elasticidade e de ruptura e o Trabalho absorvido na resistência à flexão estática da madeira na tora II sejam os mais elevados em relação às toras I e III para a maioria dos clones com a madeira na condição verde, foi na região da tora II, onde ocorreu grande parte da quebra das árvores durante o teste de resistência, com uma altura média de quebra de 2,99 m.

\section{CONCLUSÕES}

Existe diferença significativa entre os clones quanto aos parâmetros dendrométricos, sendo que as maiores variações ocorreram na variável diâmetro a altura do peito. 
O clone CNB005 apresentou, destacadamente, a maior resistência à quebra.

$\mathrm{O}$ teste de resistência das árvores à quebra não apresentou correlação quando associado às características de crescimento da árvore.

Os valores para a Densidade básica da madeira, Módulo de ruptura, Módulo de elasticidade e Trabalho absorvido à flexão estática da madeira foram maiores na madeira do clone CNB017;

$\mathrm{O}$ teste de resistência das árvores à quebra e os parâmetros físicos e mecânicos avaliados podem ser utilizados como indicadores na seleção e classificação dos clones mais tolerantes à ação dos ventos.

Estudos adicionais devem ser implementados com intuito de ampliar a base de informações que pode complementar os fatores que implicam na quebra das árvores, principalmente, a análise da forma do tronco das árvores e as práticas silviculturais adotadas;

De maneira geral, o clone mais indicado para o plantio nas áreas mais susceptíveis à ação dos ventos é o CNB017, por apresentar os melhores resultados nos parâmetros físicos e mecânicos, além de ter um valor no teste de resistência mecânica das árvores à quebra considerável.

\section{AGRADECIMENTOS}

À Empresa de Celulose Nipo Brasileira S.A. (CENIBRA), pela disponibilidade do material de estudo e pelo apoio logístico de trabalho.

\section{REFERÊNCIAS BIBLIOGRÁFICAS}

ASSOCIAÇÃO BRASILEIRA DE NORMAS TÉCNICAS. Ensaios físicos e mecânicos da madeira: MB-26/40. Rio de Janeiro: 16p.1940. ASSOCIAÇÃO BRASILEIRA DE FLORESTAS PLANTADAS - ABRAF. Anuário Estatístico da ABRAF: Ano base 2009. Brasília: 2010, $140 \mathrm{p}$.

CARDOSO, A. L. Desrama artificial de eucalipto e seu efeito na resistência a danos por vento $\mathrm{e}$ nós da madeira. $2011.94 \mathrm{f}$. Dissertação (Mestrado em Ciências Florestais) - Universidade Federal do Espírito Santo, Jerônimo Monteiro, 2011

CARUZZO, A et al. Danos provocados por ventos em florestas plantadas - Identificação de alternativas para mitigação de efeitos. WM7 Meteorologia \& Laboratório de clima e biosfera USP. Relatório Climático: Belo Oriente, 2004, 131 p.
CENIBRA. Avaliação dos danos por vento. Pesquisa e Desenvolvimento Florestal. Relatório Técnico: Belo Oriente, 2007

FERREIRA, S. Lenho de tração em Eucalyptus spp cultivados em diferentes topografias. 2007.170 f. Tese (Doutorado em Ciência e Tecnologia da Madeira) - Universidade Federal de Lavras, Lavras, 2007.

FERREIRA, S. et al. Resistência mecânica de caules de clones de Eucalyptus cultivados em diferentes topografias. Cerne, Lavras, v. 16, p. 133-140, 2010 FERREIRA, M.; KAGEYAMA, P.Y. Melhoramento genético da densidade de eucalipto. Boletim Técnico - IPEF, v. 6, n. 20, p. A1 - A15, 1978

LATORRACA, J. V.; ALBUQUERQUE, C. E. Efeito do rápido crescimento sobre as propriedades da madeira. Floresta e Ambiente, v. 7, n. 1, p.279291, 2000.

LIEGEL, L .H Assessment of hurricane rain/wind damage in Pinus caribaeae and Pinus oocarpa provenance trials in Puerto Rico. Commonwealth Forestry Review. v. 68, n.1,p.47-53, 1984

MAURI, R. Anatomia e densidade do lenho de clones de Eucalyptus urophylla $x$ Eucalyptus grandis, com variação de altitude e de topografia, no Estado de Minas Gerais. 2010.117 f. Dissertação (Mestrado em Ciências Florestais) - Universidade Federal do Espírito Santo, Jerônimo Monteiro, 2010 MELO, V. M. Variação nas produtividades da madeira de clones de Eucalyptus cultivados em diferentes topografias e sujeitas a tempestade. 2004. 115 f. Dissertação (Mestrado em Engenharia Florestal) - Universidade Federal de Lavras, Lavras, 2004.

METCALFE, C. R.; CHALK, L. Anatomy of the dicotyledons: Wood Structure and conclusion of the general introduction. 2nd ed. v. 2. New York: Oxford, 1989. $297 \mathrm{p}$.

NIKLES, D. G. et al. Genetic variation in windfirmness amongprovenances of Pinus caribaea Mor. var. hondurensis Barr. e Golf. In Queensland. In: SIMPÓSIO IUFRO EM MELHORAMENTO GENÉTICO E PRODUTIVIDADE DE ESPÉCIES FLORESTAIS DE RÁPIDO CRESCIMENTO, Águas de São Pedro, 1980. Anais... São Paulo, Sociedade Brasileira de Silvicultura, 1983. p.125-6. NIKLES, D. G. et al. Management and use of ex situ genetic resources of some tropical Acacia species in Queensland. In: TURNBULL, J. W.; CROMPTON, H. R.; PINYOPUSARERK, $\mathrm{K}$. Recent developments in acacia planting. Canberra: ACIAR, 1998. p. 184-196. 
OLIVEIRA, J. T. S. et al. Ações de ventos em povoamentos florestais. In: CHICHORRO, J. F.; GARCIA, G. O.; CALDEIRA, M. V. W.; BAUER, M. O. (Org.). Tópicos em Ciências Florestais. Visconde do Rio Branco: Suprema, 2010, p. 443-476.

RESENDE, M. D. V.; FANTINI JÚNIOR, M.

Bifurcação e quebra de copa em eucalipto: efeitos genéticos, ambientais e silviculturais. Embrapa Florestas: Colombo, 2001, 20 p. (Documento, 63) ROSADO, A. M. Avaliação da tolerância de árvores de eucalipto a quebra por vento. 2006. $43 \mathrm{f}$. Monografia (Pós-Graduação em Tecnologia de Celulose e Papel) - Universidade Federal de Viçosa, Viçosa, 2006.

SCHNEIDER, P. R; FINGER, C. A. G.; HOPPE, J. M. Efeito da intensidade de desrama na produção de Pinus Elliotti Engelm.,implantado em solo pobre, no estado do Rio Grande do Sul. Ciência Florestal, v. 9 , n. 1 , p. $35-46,1999$
SILVA, J. C.; XAVIER, B. A. Eucalipto: manual prático do fazendeiro florestal, produzindo madeira com qualidade. Viçosa, 2006. 65 p.

SILVEIRA,R.A.; MONTAGNER, L.H.; ONUKI, M. Variação de resistência a ventos em procedências de eucalyptus saligna smith na região de Guaíra, RS. Boletim de Pesquisa Florestal, Colombo, n. 13, p.1-8, dez. 1986.

SOUZA, V. R.; CARPIM, M. A.; BARRICHELO, L. E. G. Densidade básica entre procedências, classes de diâmetro e posição em arvores de Eucalyptus grandis e Eucalyptus saligna. IPEF. v. 3, p. 63-72. 1986.

THOMPSON, D. A. Effects of hurricane Allen on some jamaican forests. Commonwealth Forestry Review. v. 62, n. 2, p.107-15, 1983.

TOMAZELLO FILHO, M. Variação radial da densidade básica e da estrutura anatômica da madeira do Eucalyptus saligna e Eucalyptus grandis. IPEF. n. 29, p.37-45, 1985. 